\title{
ON THE REGULAR POINTS OF A CONTINUUM*
}

\author{
BY
}

W. L. AYRES

\section{INTRODUCTION}

1. We consider a compact, connected metric space $M$ which we shall call the continuum $M$. A point $p$ of $M$ is said to be a regular point if for each $\epsilon>0$ there exists a neighborhood $U_{p}$ of $p$ (i.e., an open subset of $M$ containing $p$ ) such that $d\left(U_{p}\right)<\epsilon$ and $F\left(U_{p}\right)$ consists of a finite number of points. The point $p$ is said to be a point of order $\alpha$ if (1) for each $\epsilon>0$ there exists a neighborhood $U_{p}$ such that $d\left(U_{p}\right)<\epsilon$ and the cardinal number of $F\left(U_{p}\right) \leqq \alpha$, and (2) $\alpha$ is the smallest cardinal number for which (1) is true. Regular points of no finite order are called points of order $\omega$. Let $M^{\alpha}$ denote the set of all points of $M$ of order $\alpha$. Then

$$
M=M^{1}+M^{2}+\cdots+M^{\omega}+M^{N_{0}}+M^{c} .
$$

These definitions were introduced by Urysohn and Menger $\dagger$ several years ago and since that time have been studied in a number of papers.

One of the interesting studies in this theory is that of the distribution and structure of the various sets $M^{\alpha}$. It is of course quite obvious that $M$ may be composed entirely of points of order 2 or entirely of points of order $c$. Urysohn $\ddagger$ has given examples to show that $M$ may be composed entirely of points of order $\omega$ or entirely of points of order $N_{0}$. Except for these four orders this is not possible, a consequence of a theorem proved independently by Urysohn, $\S \mathrm{G}$. T. Whyburn $\|$ and H. Künneth $\uparrow$ that if all points of $M$ are of order $\leqq n$, then the points of order $\leqq \frac{1}{2} n+1$ are dense in $M$.

Since $M \neq M^{n}(n \neq 2)$, it would be interesting to know more of the distribution of the points of $M^{n}$. Whyburn** has shown that $M^{n}$ is punctiform and has raised the question as to whether it is of dimension zero. In the present

* Presented to the Society, September 9, 1930; received by the editors September 9, 1930.

$\dagger$ P. Urysohn, Comptes Rendus, vol. 175 (1922), pp. 481-483; and K. Menger, Monatshefte für Mathematik und Physik, vol. 33 (1923), pp. 148-160.

$\ddagger$ Mémoire sur les multiplicités cantoriennes, 2ème Partie, Verhandelingen, Akademie van Wetenschappen, Amsterdam, vol. 13 (1927), No. 4, pp. 109-115.

\& Ibid., pp. 105-9.

\|On regular points of continua etc., Bulletin of the American Mathematical Society, vol. 35 (1929), pp. 218-224.

If Ein Theorem der Kurventheorie, Monatshefte für Mathematik und Physik, vol. 36 (1929), pp. 149-152.

** Loc. cit. 
paper we will answer this question in the affirmative as a special case of our theorem that $M^{n}+M^{n+1}+\cdots+M^{2 n-3}$ is a set of dimension zero. As corollaries of this theorem we obtain most of the previously known results concerning the distribution of the points of finite orders. In the last section of the paper we complete our study of the structure of the sets of various finite orders by examining the set $M^{2}$. Here we prove that $M^{2}$ is composed of a set of dimension zero plus a countable set of arcs.

2. Notation. Capitals will denote sets, lower case letters denote individual elements which are either points or numbers. The usual notation of the theory of sets will be employed. Below we will list some special notation which, while not new, is not universally employed by writers in this field and thus needs definition.

$p \varepsilon N \equiv p$ is an element of the set $N$.

$p$ non- $\varepsilon_{N} \equiv p$ is not an element of the set $N$.

$\rho(p, q) \equiv$ distance between the points $p$ and $q$.

$\rho(M, N) \equiv$ greatest lower bound of $\rho(p, q)$ for $p \varepsilon M$ and $q \varepsilon N$.

$d(M) \equiv$ diameter of $M \equiv$ least upper bound of $\rho(p, q)$ for $p+q \subset M$.

$S(p, \eta) \equiv$ set of all points $q$ such that $\rho(p, q)<\eta$.

$\mathcal{C}(p, N) \equiv$ component of set $N$ containing the point $p$.

$F(N) \equiv \bar{N} \cdot(M-N)+\overline{M-N} \cdot N \equiv$ frontier or boundary of $N$.

$C(N) \equiv M-N \equiv$ complement of $N$.

$\operatorname{dim}_{p} E$, $\operatorname{order}_{p} E \equiv$ dimension of set $E$ at $p$, order of $E$ at $p$.

\section{The STRUCtURE OF $M^{n}, n \neq 2$}

3. THEOREM. For any integer $n>2$, the set of all points $p$ of a continuum $M$ such that $n \leqq \operatorname{order}_{p} M \leqq 2 n-3$ is a zero-dimensional set (or vacuous).*

Let $E$ denote the set of points $p$ such that $n \leqq \operatorname{order}_{p} M \leqq 2 n-3$. Given $\epsilon>0$ and $p \mathcal{E} E$, we shall show the existence of a neighborhood $U_{p} \subset S(p, \epsilon)$ such that $F\left(U_{p}\right) \cdot E=0$, i.e., $\operatorname{dim} E=0$. If $p$ is a point of order $m$, by an order neighborhood of $p$ we shall mean a neighborhood of $p$ whose boundary consists of exactly $m$ points.

Let $Z_{1} \subset S\left(p, \frac{1}{2} \epsilon\right)$ be an order neighborhood of $p$. If $E \cdot F\left(Z_{1}\right)=0$, our proof is complete. If not, let $E \cdot F\left(Z_{1}\right)=q_{1}+q_{2}+\cdots+q_{s_{1}}\left(s_{1} \leqq 2 n-3\right)$. Let $U_{k}$ be an order neighborhood of $q_{k}\left(1 \leqq k \leqq s_{1}\right)$ such that $\bar{U}_{k} \subset S\left(q_{k}, r_{k}\right)$, where $r_{k}$ is the smaller of the numbers $\epsilon / 4$ and $\frac{1}{2} \rho\left(q_{k}, F\left(Z_{1}\right)+p-q_{k}\right)$. We see that $\bar{U}_{i} \cdot \bar{U}_{j}=0$ if $i \neq j$. Let $V_{k}=Z_{1} \cdot U_{k}$ and $W_{k}=C\left(\bar{Z}_{1}\right) \cdot U_{k}$. Since $q_{k} \varepsilon E, F\left(U_{k}\right)$

* Professor K. Menger has called attention to the fact that no use is made of the condition that $M$ be connected and compact in the proof of this theorem. Hence the theorem is true for any metric space $M$. 


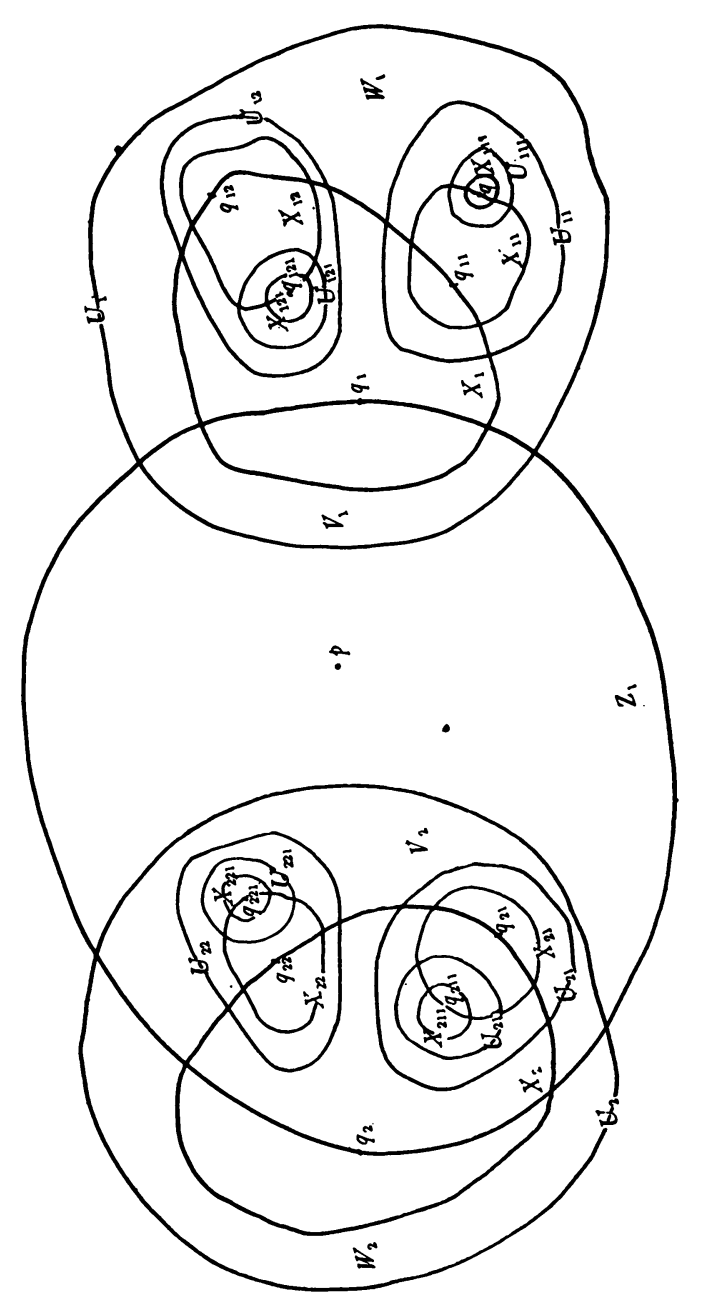


consists of $\leqq 2 n-3$ points. And since $F\left(V_{k}\right)+F\left(W_{k}\right)=F\left(U_{k}\right)+q_{k}$ and $F\left(V_{k}\right) \cdot F\left(W_{k}\right)=q_{k}$, either $F\left(V_{k}\right)$ or $F\left(W_{k}\right)$ consists of $\leqq n-1$ points. Let $I_{1}$ be the set of all integers $i$ such that $F\left(W_{i}\right)$ consists of $\leqq n-1$ points, and let $J_{1}$ be the set of all integers $1 \leqq j \leqq s_{1}$ not in $I_{1}$. Let $X_{k}$ be an order neighborhood of $q_{k}$ such that $\bar{X}_{k} \subset U_{k}$. Now let

$$
Z_{2}=Z_{1}+\sum X_{i}-\sum \bar{X}_{i},
$$

where the first summation extends over $I_{1}$ and the second over $J_{1}$.

If $F\left(Z_{2}\right) \cdot E=0$, then $Z_{2}$ is the desired neighborhood $U_{p}$. If not, we have $F\left(Z_{2}\right) \cdot E \subset \sum_{k} F\left(X_{k}\right)$. Let $F\left(X_{k}\right) \cdot F\left(Z_{2}\right) \cdot E=q_{k 1}+q_{k 2}+\cdots+q_{k s_{2} k}$. For each point $q_{i m}\left(i \varepsilon_{1}\right)$, let $U_{i m}$ be an order neighborhood of $q_{i m}$ such that $\bar{U}_{i m} \subset W_{i}$ - $S\left(q_{i m}, \boldsymbol{r}_{i m}\right)$, where $\boldsymbol{r}_{i m}$ is the smaller of the numbers $\epsilon / 8$ and $\frac{1}{2} \rho\left(q_{i m}, F\left(X_{i}\right)\right.$ $\left.-q_{i m}\right)$. For each point $q_{j m}\left(j \varepsilon_{1}\right)$, let $U_{j m}$ be an order neighborhood of $q_{j m}$ such that $\bar{U}_{j m} \subset V_{j m} \cdot S\left(q_{j m}, r_{j m}\right)$, where $r_{j m}$ is the smaller of the numbers $\epsilon / 8$ and $\frac{1}{2} \rho\left(q_{j m}, F\left(X_{j}\right)-q_{j m}\right)$. We see that $\bar{U}_{k_{1} m_{1}} \cdot \bar{U}_{k_{2} m_{2}}=0$ unless $k_{1}=k_{2}$, $m_{1}=m_{2}$. Let $V_{k m}=Z_{2} \cdot U_{k m}$ and $W_{k m}=C\left(\bar{Z}_{2}\right) \cdot U_{k m}$. Since $q_{k m} \varepsilon E, F\left(U_{k m}\right)$ consists of $\leqq 2 n-3$ points. Then either $F\left(W_{k m}\right)$ or $F\left(V_{k m}\right)$ consists of $\leqq n-1$ points. Let $I_{2}$ be the set of all pairs $(k, m)$ such that $F\left(W_{k m}\right)$ consists of $\leqq n-1$ points. Let $J_{2}$ be the set of all pairs $(k, m)$ for which $U_{k m}$ is defined that are not in $I_{2}$. Let $X_{k m}$ be an order neighborhood of $q_{k m}$ such that $\bar{X}_{k m} \subset U_{k m}$. Now let

$$
Z_{3}=Z_{2}+\sum X_{k m}-\sum \bar{X}_{k m},
$$

where in the first summation $(k, m) \varepsilon_{2}$ and in the second $(k, m) \varepsilon J_{2}$.

If $F\left(Z_{3}\right) \cdot E=0, Z_{3}$ is the desired neighborhood $U_{p}$. If not, we repeat this process on the points of $F\left(Z_{3}\right) \cdot E=F\left(Z_{3}\right) \cdot E \cdot \sum_{k} \sum_{m} F\left(X_{k m}\right)$. Continuing this process, at some stage we reach a neighborhood $Z_{i}$ such that $F\left(Z_{i}\right) \cdot E=0$ or the process continues indefinitely.

In case the process continues indefinitely, we define a monotonic increasing sequence of neighborhoods of $p$ as follows:

$$
Y_{1}=Z_{1}-\sum_{k=1}^{s_{1}} \bar{U}_{k}, \quad Y_{2}=Z_{2}-\sum_{k=1}^{s_{1}} \sum_{m=1}^{s_{2} k} \bar{U}_{k m},
$$

and similarly we define $Y_{t}$ for each positive integer $t$. Now let

$$
U_{p}=\sum_{t=1}^{\infty} Y_{t}
$$

Since $U_{p} \subset Z_{1}+\sum_{k} U_{k} \subset S(p, \epsilon)$, then $U_{p}$ is the desired neighborhood if $F\left(U_{p}\right) \cdot E=0$. Every point of $F\left(Z_{t}\right)$ not belonging to $E$ is a point of $F\left(Y_{t}\right)$ and of every $F\left(Y_{\imath}\right)$ for $s \geqq t$. Then the points of $F\left(U_{p}\right)$ are of two classes: 
(a) points which belong to $F\left(Y_{s}\right)$ for every $s$ greater than some fixed integer, and (b) $\Pi_{t=1}^{\infty}\left(F\left(U_{p}\right)-F\left(Y_{t}\right)\right)$. Just above it was seen that no point of the class (a) is a point of $E$. Now as

$$
F\left(U_{p}\right)-F\left(Y_{t}\right) \subset \sum U_{k_{1} k_{2} \cdots k_{t}} \text {, }
$$

we have that every point of $F\left(U_{p}\right)$ of class (b) belongs to the set

$$
H=\prod_{t=1}^{\infty} \sum U_{k_{1} k_{2} \cdots k_{t}} .
$$

Now consider any point $y$ of $H$. Each neighborhood $U_{k m}$ is a subset of $V_{k}$ or $W_{k}$ according as $k \varepsilon I_{1}$ or $J_{1}$, i.e. $U_{k m}$ is a subset of a neighborhood of the first stage whose boundary contains $\leqq n-1$ points. Similarly for the neighborhoods $U_{k_{1} k_{2}} \cdots_{k_{t}}$ of any stage. Then at each stage $y$ belongs to a neighborhood whose boundary contains $\leqq n-1$ points. And as the diameters of the neighborhoods approach zero, it follows that $\operatorname{order}_{y} M \leqq n-1$. Hence $y$ non- $\varepsilon E$. Then $U_{p}$ is the desired neighborhood of $p$ as

$$
U_{p} \subset S(p, \epsilon) \text { and } F\left(U_{p}\right) \cdot E=0 .
$$

4. This section proves corollaries to the preceding theorem.

CoROllary 1. For each positive integer $n \neq 2$, the set of all points of $M$ of order $n$ is zero-dimensional (or vacuous).

Since a subset of a vacuous or zero-dimensional set is necessarily of the same type, for $n>2$ this follows from our theorem. That the set $M^{1}$ is of this type has been shown by Menger and Urysohn.*

COROLlary $2 . \dagger$ There exists no continuum all of whose points are of order $n \neq 2$.

If $M$ is a continuum, $\operatorname{dim} M \geqq 1$. Hence $M-M^{n} \neq 0$ for any $n \neq 2$.

CoRollary 3. The simple closed curve is the only (compact) continuum all of whose points are of the same finite order.

COROLLARY 4. If the order of every point of the continuum $M$ is $\leqq m$, then the points of order $\leqq \frac{1}{2} m+1$ are dense in $M$.

From our theorem it follows that the set of all points $p$ such that $\frac{1}{2} m+1<\operatorname{order}_{p} M \leqq m$ is zero-dimensional. Hence it contains no open subset and the remaining points are dense in $M$.

5. Remarks. It may be noticed in the proof of the preceding theorem

* K. Menger, Mathematische Annalen, vol. 95 (1925), p. 293; and P. Urysohn, Second reference, p. 79.

$\dagger$ The corollaries 2, 3 and 4 are all known results. See the papers cited in the Introduction. 
that, while at each stage the neighborhoods have only finite boundaries, the final neighborhood $U_{p}$ may have an infinite boundary. This raises the question as to whether it is possible to select $U_{p}$ with only a finite boundary. This is not always true. If we join two of the Sierpinski triangle curves* at the vertices we have a continuum containing only points of orders 3 and 4 . Now if we take $n=4$ the only neighborhoods $U_{p}$ such that $F\left(U_{p}\right) \cdot E=0$ have infinite boundaries.

We have seen that $\operatorname{dim} M^{n}=0$ for $n>2$. It would be interesting now to determine the conditions under which $\operatorname{dim} \sum_{n>2} M^{n}=0$.

Urysohn has constructed very interesting examples of continua containing points of orders $n$ and $2 n-2$ only for any $n>2$. This should lead to a study of continua containing points of orders $m$ and $n$ only $(m>n>2)$. From our theorem or Corollary 4 , it follows that such a continuum can exist only if $m \geqq 2 n-2$. It would be interesting to determine whether it can exist if $m \neq k(n-1)$. Also it seems likely that in such a continuum the points of order $m$ are countable.

\section{The STRUCTURE OF $M^{2}$}

6. Lemma. If $p$ is a point of $M^{2}$ and a cut point im kleinen of $M$ and $\operatorname{dim}_{p}$ $M^{2}>0$, then $M$ contains an arc one of whose end points is $p$ such that the arcsegment $\dagger$ is an open subset of $M$.

As $p$ is a cut point im kleinen of $M$, there is a neighborhood $Z_{p}$ such that $\mathcal{C}\left(p, \bar{Z}_{p}\right)=R+S$, where $R$ and $S$ are continua and $R \cdot S=p$. Then either $\operatorname{dim}_{p} R \cdot M^{2}>0$ or $\operatorname{dim}_{p} S \cdot M^{2}>0$ and we suppose the former. There exists a neighborhood $U_{p}$ such that if the neighborhood $V_{p} \subset U_{p}$, then

$$
F\left(V_{p}\right) \cdot M^{2} \cdot R \neq 0 \text {. }
$$

As $p \varepsilon M^{2}$ there is a neighborhood $W_{p}$ such that $\bar{W}_{p} \subset U_{p}$ and $F\left(W_{p}\right) \cdot R$ is a single point $r$ and $R-\bar{W}_{p} \neq 0$. Let $G=\bar{W}_{p} \cdot R$. As $r \varepsilon M^{2}$ and $R-\bar{W}_{p} \neq 0$, the point $r \varepsilon G^{1}$, i.e. the points of $G$ of order 1 with respect to $G$. As $p \mathcal{E} G^{1}$ and $M^{2} \cdot G=G^{2}+p+r$, we will complete our proof by establishing the next lemma.

7. Lemma. If $G$ is a continuum and (a) $p$ and $r$ are end points of $G$, and (b) for any neighborhood $U_{p}$ such that $r$ non- $\varepsilon \bar{U}_{p}$, the set $F\left(U_{p}\right) \cdot G^{2} \neq 0$, then $G$ is an arc with end points $p$ and $r$.

Let $Q$ denote the set consisting of $p+r+$ those points of $G^{2}$ which separate $p$ and $r$ in $G$. We shall prove that $Q$ is a closed set. Let $\left[q_{i}\right]$ be a sequence of

* Comptes Rendus, vol. 160 (1915), pp. 302-5; and Prace Matematyczno-Fizyczne, vol. 27 (1915), pp. 77-86.

† By the arc-segment is meant the arc minus its end points. 
distinct points of $Q$ such that $\lim q_{i}=q$. We shall show that $q \mathcal{E} Q$. This is true if $p=q$ or $r=q$. If $p \neq q \neq r$, there are two cases:

Case I. There is an infinite subsequence $\left[q_{i_{k}}\right]$ such that

$$
\begin{gathered}
G-q_{i_{k}}=G_{p k}+G_{r k}, G_{p k} \cdot \bar{G}_{r k}+\bar{G}_{p k} \cdot G_{r k}=0, \\
p \varepsilon G_{p k}, q+q_{i_{k+1}}+r \subset G_{r k} .
\end{gathered}
$$

Since $q_{i_{k}} \varepsilon G^{2}, G_{p k}$ and $G_{r k}$ are connected and $G_{p k} \subset G_{p k+1}$. Let

$$
X_{p}=\sum_{k=1}^{\infty} G_{p k}
$$

The set $X_{p}$ is a neighborhood of $p^{*}$ and $q$ non- $\varepsilon X_{p}$. Suppose $F\left(X_{p}\right) \supset t \varepsilon G^{2}+r$, $t \neq q$. Let $\left[t_{n}\right]$ be a sequence such that $t_{n} \varepsilon G_{p n}, \lim t_{n}=t$. As $t \varepsilon \Pi G_{r k}$ it follows that if $N$ is any subcontinuum of $G$ containing $t$ and $t_{n}$, then $q_{i j} \varepsilon N$ for $j \geqq n$. Then $N$ contains $q$. For this reason $G$ is not locally connected at $t$. But $t \varepsilon G^{2}+r$ and a continuum is locally connected at every point of finite order. Hence $t$ cannot exist. By condition (b), $F\left(X_{p}\right) \cdot G^{2} \neq 0$. Then $q \varepsilon G^{2}$.

Suppose there exists a neighborhood $U_{q}$ of $q$ such that both $p$ and $r$ belong to one component $L$ of $G-U_{q}$. Then $L \supset \sum q_{i_{k}}$. But as $\lim q_{i_{k}}=q$, for $k$ sufficiently large $q_{i_{k}} \subset U_{q}$. But this is absurd as $L \cdot U_{q}=0$. Hence, for any neighborhood $U_{q}$ such that $p+r \subset G-U_{q}, p$ and $r$ belong to different components of $G-U_{q}$. Now let $\left[U_{n}\right]$ be a set of neighborhoods of $q$ such that $\bar{U}_{n+1} \subset U_{n}, d\left(U_{n}\right)<1 / n, F\left(U_{n}\right)$ consists of two points, $G-U_{1} \supset p+r$. It is easily seen that

$$
G-U_{n}=\mathcal{C}\left(p, G-U_{n}\right)+\mathcal{C}\left(r, G-U_{n}\right)
$$

and thus

Then

$$
\begin{aligned}
& \mathcal{C}\left(p, G-U_{n+1}\right)>\mathcal{C}\left(p, G-U_{n}\right), \\
& \mathcal{C}\left(r, G-U_{n+1}\right)>\mathcal{C}\left(r, G-U_{n}\right) .
\end{aligned}
$$

Hence $q \mathcal{E} Q$.

$$
G-q=\sum_{n} \mathcal{C}\left(p, G-U_{n}\right)+\sum_{n} \mathrm{C}\left(r, G-U_{n}\right) \text {. }
$$

Case II. There is an infinite subsequence $\left[q_{i_{k}}\right]$ such that

Let

$$
\begin{gathered}
G-q_{i_{k}}=G_{p k}+G_{r k}, \bar{G}_{p k} \cdot G_{r k}+G_{p k} \cdot \bar{G}_{r k}=0, \\
p+q+q_{i_{k+1}} \subset G_{p k}, r \varepsilon_{G_{r k}} .
\end{gathered}
$$

$$
P=\sum_{k=1}^{\infty} G_{r k} .
$$

* For this lemma we consider $G$ as our space. Relative to $G$ alone, the set $X_{p}$ is open and hence is a neighborhood. 
Exactly as in Case I we may show that if $F(P) \supset t \in G^{2}+p, t \neq q$, then $G$ is not locally connected at $t$. We have thus

$$
F(P) \cdot\left(G^{2}-q+p\right)=0 .
$$

The set $G-\bar{P}$ is a neighborhood of $p$, and $r$ non- $\mathcal{E} F(G-\bar{P})$. We have $F(G-\bar{P}) \subset F(P)$. From (1) then,

$$
F(G-\bar{P}) \cdot\left(G^{2}-q\right)=0 ;
$$

and, from condition (b), we must have $q \mathcal{E} G^{2}$. As in Case I we may show that $q$ separates $p$ and $r$ in $G$.

We have shown that the set $Q$ is closed. Let $H$ be a subcontinuum of $G$ irreducible between $p$ and $r$. Suppose there exists a point $x \varepsilon H-Q$, and let $G_{x}=\mathcal{C}(x, H-Q)$. Let $y \varepsilon \bar{G}_{x} \cdot Q$. Since $p \varepsilon G^{1}$, there is a neighborhood $Z_{p}$ such that $F\left(Z_{p}\right)=u$ (a single point) and $(x+r) \cdot \bar{Z}_{p}=0$. The point $u$ separates $p$ and $r$, and $u \in Q$ from condition (b). Then $G_{x} \subset G-\bar{Z}_{p}$ and hence $y \neq p$. Similarly $y \neq r$. Let $U_{y}$ be a neighborhood of $y$ such that $(x+p+r) \cdot \bar{U}_{y}=0$. As $y \in G^{2}$ there is a neighborhood $V_{y} \subset U_{y}$ such that $F\left(V_{y}\right)=u_{1}+u_{2}$. From the fact that $H$ is an irreducible continuum it follows that both $u_{1}$ and $u_{2}$ separate $p$ and $r$ in $G$. Then there exists a neighborhood $Y_{p i}(i=1,2)$ of $p$ such that $F\left(Y_{p i}\right)=u_{i}$ and thus $u_{i} \varepsilon Q$ from (b). The connected set $G_{x}$ contains a point in $V_{y}$ and a point $x$ non- $\varepsilon V_{y}$, so either $u_{1}$ or $u_{2}$ belongs to $G_{x}$. But $G_{x} \cdot Q=0$ and $u_{i} \varepsilon Q$. This is a contradiction and hence $H-Q=0$.

From this it follows that $H=Q=G$. Then $p+r=G^{1}$ and $G-p-r=G^{2}$. By a result due to Urysohn and Menger* the set $G$ is an arc with end points $p$ and $r$.

8. Lemma. If $p \varepsilon M^{2}$ and $\operatorname{dim}_{p} M^{2}>0$, then $p$ is a cut point im kleinen of $M$.

There exists a neighborhood $U_{p}$ such that if $V_{p} \subset U_{p}$ then

$$
F\left(V_{p}\right) \cdot M^{2} \neq 0
$$

and

$$
F\left(V_{p}\right) \supset \text { at least two points. }
$$

There exists a sequence of neighborhoods $V_{1}, V_{2}, \cdots$ of $p$ such that $V_{i} \subset U_{p}$, $\bar{V}_{i+1} \subset V_{i}, d\left(V_{i}\right)<1 / i, F\left(V_{i}\right)=u_{i}+v_{i}$. Consider the set $\bar{V}_{i-1}-V_{i}$. Obviously the set $u_{i-1}+v_{i-1}+u_{i}+v_{i}=G \subset \bar{V}_{i-1}-V_{i}$. Let $x \in \bar{V}_{i-1}-V_{i}$. Then $\mathcal{C}\left(x, \bar{V}_{i-1}\right.$ $\left.-V_{i}\right) \cdot G \neq 0$, for otherwise there would be a separation of $\bar{V}_{i-1}-V_{i}$ into mutually separated sets containing $\mathcal{C}\left(x, \bar{V}_{i-1}-V_{i}\right)$ and $G$ respectively. But this separation would effect a separation of $M$, which was connected. Then $\bar{V}_{i-1}-V_{i}$ contains at most four components. But $\mathcal{C}\left(u_{i}, \bar{V}_{i-1}-V_{i}\right)$ must con-

* P. Urysohn, loc. cit., and K. Menger, loc. cit., p. 303. 
tain $u_{i-1}$ or $v_{i-1}$ for otherwise $V_{i}+\mathcal{C}\left(u_{i}, \bar{V}_{i-1}-V_{i}\right)$ is a neighborhood $V_{p} \subset U_{p}$, but $F\left(V_{p}\right)=v_{i}$ contrary to (3). Similarly for $\mathcal{C}\left(v_{i}, \bar{V}_{i-1}-V_{i}\right)$. Also $\mathcal{C}\left(u_{i-1}\right.$, $\left.\bar{V}_{i-1}-V_{i}\right) \cdot\left(u_{i}+v_{i}\right) \neq 0$, for otherwise $V_{i-1}-\mathcal{C}\left(u_{i-1}, \bar{V}_{i-1}-V_{i}\right)$ is a neighborhood $V_{p}$ and (3) is not true. Similarly for $\mathcal{C}\left(v_{i-1}, \bar{V}_{i-1}-V_{i}\right)$. Thus $\bar{V}_{i-1}-V_{i}$ has at most two components and each contains a point of $u_{i}+v_{i}$ and a point of $u_{i-1}+v_{i-1}$. From (2) we have that $u_{i} \varepsilon M^{2}$ or $v_{i} \varepsilon M^{2}$. Further at least one of these points is a point of $M^{2}$ and is such that $\operatorname{dim} M^{2} \cdot\left(\bar{V}_{i-1}-V_{i}\right)>0$ at the point. For suppose $u_{i}+v_{i} \subset M^{2}, \operatorname{dim} M^{2} \cdot\left(\bar{V}_{i-1}-V_{i}\right)=0$ at both points. There exists a neighborhood $U_{u_{i}} \subset V_{i-1}$ such that $F\left(U_{u_{i}}\right) \cdot M^{2} \cdot\left(\bar{V}_{i-1}-V_{i}\right)=0$, and similarly a neighborhood $U_{v_{i}} \subset V_{i-1}$. Then $W_{p}=V_{i}+U_{u_{i}}+U_{v_{i}}$ is a neighborhood of $p$ such that $W_{p} \subset U_{p}$ and $F\left(W_{p}\right) \cdot M^{2}=0$ contrary to (2). In case $u_{i} \varepsilon M^{2}, v_{i}$ non- $\varepsilon M^{2}, \operatorname{dim}_{u_{i}} M^{2} \cdot\left(\bar{V}_{i-1}-V_{i}\right)=0$, we define $W_{p}=V_{i}+U_{u_{i}}$. The only other possibilities are the interchange of $u_{i}$ and $v_{i}$.

Case I. Suppose (a) $u_{i} \varepsilon M^{2}$, (b) $\operatorname{dim}_{u_{i}} M^{2} \cdot\left(\bar{V}_{i-1}-V_{i}\right)>0$, (c) $v_{i}$ non- $\varepsilon M^{2}$. Since $u_{i} \varepsilon_{M} M^{2}$ there is a neighborhood $U_{u_{i}} \subset V_{i-1}-\bar{V}_{i+1}-v_{i}$ such that $F\left(U_{u_{i}}\right)=x+y$. Since there is a component of $\bar{V}_{i}-V_{i+1}$ containing $u_{i}$ and a point of $u_{i+1}+v_{i+1}$, either $x$ or $y$, let us suppose $y$, belongs to $V_{i}-\vec{V}_{i+1}$. Then $x \mathcal{E}^{2}$, since $\operatorname{dim}_{u_{i}} M^{2} \cdot\left(\bar{V}_{i-1}-V_{i}\right)>0$. And $\mathcal{C}\left(x, \bar{U}_{u_{i}} \cdot\left(\bar{V}_{i-1}-V_{i}\right)\right)=H$ is a continuum such that $u_{i}+x \subset H^{1}$. Now if $W_{u_{i}}$ is any neighborhood of $u_{i}$ such that $x$ non- $\varepsilon \bar{W}_{u_{i}}$, then $W_{u_{i}} \cdot U_{u_{i}}$ is a neighborhood such that $F\left(W_{u_{i}} \cdot U_{u_{i}}\right)$ . $\left(\bar{V}_{i-1}-V_{i}\right) \subset F\left(W_{u_{i}}\right) \cdot H$. Then from (b) we have that $F\left(W_{u_{i}}\right) \cdot H \cdot M^{2} \neq 0$. Thus by the lemma of $\S 7$ the continuum $H$ is an $\operatorname{arc}$ from $x$ to $u_{i}$. Now let $N_{i}$ denote $u_{i}$ plus all points of $\bar{V}_{i-1}-V_{i}$ that can be joined to $u_{i}$ by an open arc-segment of $\bar{V}_{i-1}-V_{i}$, i.e., a point $z \varepsilon \bar{V}_{i-1}-V_{i}$ is a point of $N_{i}$ if there is an arc $A \subset \bar{V}_{i-1}-V_{i}$ with end points $z$ and $u_{i}$ such that $M-A+u_{i}+z$ is closed. Evidently $H \subset N_{i}$. Since $u_{i}$ is a point of order 1 of $\bar{V}_{i-1}-V_{i}$, we see that $N_{i}$ is an arc or homeomorphic with an arc minus one end point. Consider the second possibility. As $V_{i}+N_{i}$ is a neighborhood of $p$ contained in $U_{p}$, it follows from (2) and (c) that one point of $\bar{N}_{i}-N_{i}$ is a point $q \varepsilon M^{2}$. Since $M$ is locally connected at $q$ we have that $q$ is the only point of $\bar{N}_{i}-N_{i}$. Then $N_{i}+q$ is an arc and $q$ may be joined to $u_{i}$ by the open arc-segment $N_{i}-u_{i}$. Thus $q \varepsilon N_{i}$ which is a contradiction. Hence $N_{i}$ is an arc and let $u_{i}$ and $q$ be its end points. As $V_{i}+N_{i}-q$ is a neighborhood of $p, q \varepsilon_{M^{2}}$ from (2) and (c). If $q$ non- $\varepsilon_{u_{i-1}}+v_{i-1}$, there exists a neighborhood $U_{q} \subset V_{i-1}-\bar{V}_{i}$ such that $F\left(U_{q}\right)$ consists of just two points. Then just as was the case with $H$, we may show that $\bar{U}_{q}-N_{i}+q$ is an arc by using the lemma of $\S 7$. Then any point of this new arc belongs to $N_{i}$ by definition, a contradiction.

Hence $q \varepsilon_{u_{i-1}+v_{i-1}}$, say $q=u_{i-1}$. As $q \varepsilon_{M^{2}}$ and $q$ is a limit point of $M-\bar{V}_{i-1}, q=u_{i-1}$ is a point of order 1 of $\bar{V}_{i-1}-V_{i}$. Hence $N_{i}$ is a component of $\bar{V}_{i-1}-V_{i}$ and $\bar{V}_{i-1}-V_{i}-N_{i}$ is closed. Then 


$$
\bar{V}_{i-1}-V_{i}=N_{i}+M_{i}
$$

where $M_{i}$ is a continuum containing $v_{i}$ and $v_{i-1}$.

Case II. Suppose (a) $u_{i}+v_{i} \subset M^{2}$, (b) $\operatorname{dim}_{u_{i}} M^{2} \cdot\left(\bar{V}_{i-1}-V_{i}\right)>0$, (c) $\operatorname{dim}_{v_{i}}$ $M^{2} \cdot\left(\bar{V}_{i-1}-V_{i}\right)=0$. From (c) there exists a neighborhood $U_{v_{i}}$ such that $\bar{U}_{v_{i}} \subset V_{i-1}-\bar{V}_{i+1}-u_{i}$ and $F\left(U_{v_{i}}\right) \cdot\left(\bar{V}_{i-1}-V_{i}\right) \cdot M^{2}=0$. The proof in Case II is exactly the same as Case I except that where a neighborhood of $p$ is formed by taking $V_{i}$ plus some open set, we take $V_{i}+U_{v_{i}}$ plus the open set.

Case III. Suppose (a) $u_{i}+v_{i} \subset M^{2}$, (b) $\operatorname{dim}_{u_{i}} M^{2} \cdot\left(\bar{V}_{i-1}-V_{i}\right)>0$, (c) $\operatorname{dim}_{v_{i}} M^{2} \cdot\left(\bar{V}_{i-1}-V_{i}\right)>0$. Let $N_{u_{i}}$ and $N_{v_{i}}$ denote the sets consisting of $u_{i}$ and $v_{i}$ respectively together with all points of $\bar{V}_{i-1}-V_{i}$ that can be joined to $u_{i}$ or $v_{i}$, as the case may be, by an arc of $\bar{V}_{i-1}-V_{i}$ such that the arc-segment is an open subset of $M$. The sets $N_{u_{i}}$ and $N_{v_{i}}$ are either arcs or homeomorphic with an arc minus one end point. Either $N_{u_{i}}$ or $N_{v_{i}}$ is an arc, for otherwise $V_{i}+N_{u_{i}}+N_{v_{i}}$ is a neighborhood of $p$ and we have a contradiction exactly as in Case I. Also we may prove, similar to the proof of Case I, that one of these must be an arc with $u_{i-1}$ or $v_{i-1}$ as one end point and this arc is a component of $\bar{V}_{i-1}-V_{i}$ and also an open subset of it. Hence

$$
\bar{V}_{i-1}-V_{i}=N_{i}+M_{i}, N_{i} \cdot M_{i}=0,
$$

where each is a continuum joining a point of $u_{i}+v_{i}$ to a point of $u_{i-1}+v_{i-1}$.

Thus we have seen in any case that each set $\bar{V}_{i-1}-V_{i}$ consists of two components $N_{i}$ and $M_{i}$, and suppose the components are so lettered that $N_{i} \cdot N_{i+1} \neq 0 \neq M_{i} \cdot M_{i+1}$. Then

$$
\bar{V}_{1}=\left(p+\sum_{i=2}^{\infty} N_{i}\right)+\left(p+\sum_{i=2}^{\infty} M_{i}\right)
$$

is the sum of two continua having only $p$ in common. Hence $p$ is a cut point im kleinen of $M$.

9. TheOREM. If $M$ is any continuum, then $M^{2}=H+K$, where (a) $H$ is vacuous or $\operatorname{dim} H=0$, (b) if $p \mathcal{E} H$ then $\operatorname{dim}_{p} M^{2}=0$, (c) $K$ is vacuous or a countable set of arcs $A_{i}$, (d) each arc-segment $A_{i}$ is an open subset of $M$, (e) $A_{i} \cdot A_{j}=0, A_{i} \subset A_{i}$, or $A_{j} \subset A_{i}$.

Let $K$ denote the set of all points $p$ such that $p \varepsilon M^{2}$ and $\operatorname{dim}_{p} M^{2}>0$. Then if $q \varepsilon M^{2}-K, \operatorname{dim}_{q} M^{2}=0$. Then as $H=M^{2}-K \subset M^{2}, \operatorname{dim}_{q} H=0$ for each $q \varepsilon H$. Now let $p \varepsilon K$. By the lemmas of $\$ \$ 6$ and 8 , there is an arc $B_{p}$ one of whose end points is $p$ such that $B_{p} \subset M^{2}$ and $\left\langle B_{p}\right\rangle$, i.e. $B_{p}$ minus its end points, is an open subset of $M$. And the $\operatorname{arcs} B_{p}$ may be chosen so that if $x$ is any point of an open arc-segment of $M$, there is some point $p \varepsilon K$ 
such that $x \mathcal{\varepsilon}\left\langle B_{p}\right\rangle$. Then the set $\left[\left\langle B_{p}\right\rangle\right]$ for all points $p \varepsilon K$ is a set of open subsets of $M$ covering all points of open arc-segments of $M$. By the Lindelöf property there is a countable subset, $\left\langle B_{1}\right\rangle,\left\langle B_{2}\right\rangle, \cdots$, which covers the same set. Since each $B_{i} \subset M^{2}$ it follows that if the sum of any finite number of the $B_{1}$ 's is connected, then it is an arc. From this we find that

$$
N=\sum_{i} B_{i}
$$

consists of a countable number of maximal connęcted subsets, $N_{1}, N_{2}, \cdots$, each of which is homeomorphic with a closed, half-open, or an open interval. Take the interval $(0,1)$ and let $\Phi_{i}$ be the homeomorphism which carries this interval (closed, half-open, or open) into $N_{i}$. There are three cases.

Case I. $N_{i}$ is homeomorphic with the closed interval. In this case $N_{i}$ is an arc and let $A_{i 1}=N_{i}, A_{i j}=0$ for $j>1$.

Case II. $N_{i}$ is homeomorphic with the half-open interval $(0,1>$. In case $\lim _{n \rightarrow \infty} \Phi_{i}(n /(n+1))$ exists and is a point $x_{i} \varepsilon M^{2}$, then $N_{i}+x_{i}$ is an arc c $M^{2}$ and we define $A_{i 1}=N_{i}+x_{i}, A_{i j}=0$ for $j>1$. In case the limit does not exist or $x_{i}$ non- $\varepsilon M^{2}$, we define $A_{i j}=\Phi_{i}\left(I_{j}\right)$, where $I_{j}$ is the closed interval $(0, j /(j+1))$.

Case III. $N_{i}$ is homeomorphic with the open interval $\langle 0,1\rangle$. Suppose (a) $\lim _{n \rightarrow \infty} \Phi_{i}(1 / n)$ exists and is a point $x_{i} \varepsilon M^{2}$, (b) $\lim _{n \rightarrow \infty} \Phi_{i}(n /(n+1))$ exists and is a point $y_{i} \varepsilon M^{2}$. Then $N_{i}+x_{i}+y_{i}$ is an arc $\subset M^{2}$ and we define $A_{i 1}=N_{i}+x_{i}+y_{i}, A_{i j}=0$ for $j>1$. If (a) is true and (b) false, we define $A_{i j}=x_{i}+\Phi_{i}\left(I_{j}\right)$, where $I_{j}$ is the half-open interval $\left.<0, j /(j+1)\right)$. If (b) is true and (a) false, we define $A_{i j}=y_{i}+\Phi_{i}\left(I_{j}\right)$, where $I_{j}$ is the half-open interval $\left(1 /(j+1), 1>\right.$. If both (a) and (b) are false, we define $A_{i j}=\Phi_{i}\left(I_{j}\right)$, where $I_{j}$ is the closed interval $(1 /(j+2),(j+1) /(j+2))$.

We shall show now that $\left[A_{i j}\right]$ is the required countable set of arcs, i.e., for every $i$ and $j, A_{i j} \subset K$, and if $p \varepsilon K$, then $p \varepsilon_{A_{i j}}$ for some $i$ and $j$. The first part is obvious from the definition of $A_{i j}$. Now if $p \varepsilon K$, there is an arc $B_{p} \subset M^{2}$ with $p$ as one end point such that $\left\langle B_{p}\right\rangle$ is an open subset of $M$. Now as $\left[B_{i}\right]$ covers all such open subsets, there exists an integer $i$ such that $<B_{p}>c N_{i}$. If $p \varepsilon N_{i}$, then $p \varepsilon_{A_{i j}}$ for some $j$. If $p$ non- $\varepsilon N_{i}$, since $p$ is an end point of $B_{p}$, it follows that either $\lim _{n \rightarrow \infty} \Phi_{i}(1 / n)$ or $\lim _{n \rightarrow \infty} \Phi_{i}(n /(n+1))$ exists and is the point $p$. In this case $p \mathcal{E}_{A_{i 1} \text {. }}$

UNIVERSity OF Michigan, ANN ARbor, Mich. 